\title{
Slowly developing toxic epidermal necrolysis-like reaction associated with pemetrexed and carboplatin
}

\author{
Gerhard Eichhoff \\ Capital and Coast District Health Board, Dermatology Service, Wellington Hospital, Wellington, New Zealand
}

\begin{abstract}
Most cutaneous adverse drug reactions reported in association with chemotherapy, such as a limited maculopapular rash, are considered mild and do not affect the continuation of the treatment. Toxic epidermal necrolysis (TEN), however, is a life-threatening reaction that needs treatment discontinuation. The present case shows the slow progression from a pemetrexed and carboplatin-associated maculopapular rash to a TEN-like reaction.
\end{abstract}

Keywords: toxic epidermal necrolysis, cutaneous adverse drug reactions, drug rash, pemetrexed

\section{Introduction}

Toxic epidermal necrolysis is a serious adverse drug reaction (SADR) linked to high morbidity and mortality. The usual course of toxic epidermal necrolysis (TEN) is a rapid progression from an erythema to epidermolysis [1]. The multitargeted antifolate pemetrexed is frequently associated with maculopapular rash and is a known elicitor for TEN, whereas the combination with carbo or cisplatin does not substantially increase the risk for cutaneous adverse events [2, 3].

\section{Clinical case}

A 70-year-old female patient without other relevant medical history was started on pemetrexed and carboplatin for mesothelioma after vitamin B12 and folic acid supplementation. The patient developed a rash on the anterior chest wall 7 days after the first cycle which persisted despite treatment with loratadine. Four days after the second cycle (14 days after the onset of the rash), the patient was admitted with widespread maculopapular eruptions treated with betamethasone valerate $0.1 \%$ cream along with cetirizine $10 \mathrm{mg}$ twice daily. The patient's additional symptoms were fever, pancytopenia, including neutropenia, seizures and confusion, pulmonary embolism, and nausea. Over the next few days, the rash continued to coalesce and spread. Forty days after the initial onset, $80 \%$ of the total body's surface area (TBSA) was affected with beginning denudation (Figure 1). Histology showed widespread epidermal necrosis with multiple dyskeratotic keratinocytes. A TEN-like reaction was diagnosed. Treatment with prednisone $50 \mathrm{mg}$
Correspondence to: Gerhard Eichhoff Email: gerhard.eichhoff@ccdhb.org.nz

ecancer 2020, 14:1010

https://doi.org/10.3332/ecancer.2020.1010

Published: $13 / 02 / 2020$

Received: 11/11/2019

Publication costs for this article were supported by ecancer (UK Charity number 1176307).

Copyright: (c) the authors; licensee ecancermedicalscience. This is an Open Access article distributed under the terms of the Creative Commons Attribution License (http:// creativecommons.org/licenses/by/3.0), which permits unrestricted use, distribution, and reproduction in any medium, provided the original work is properly cited. 

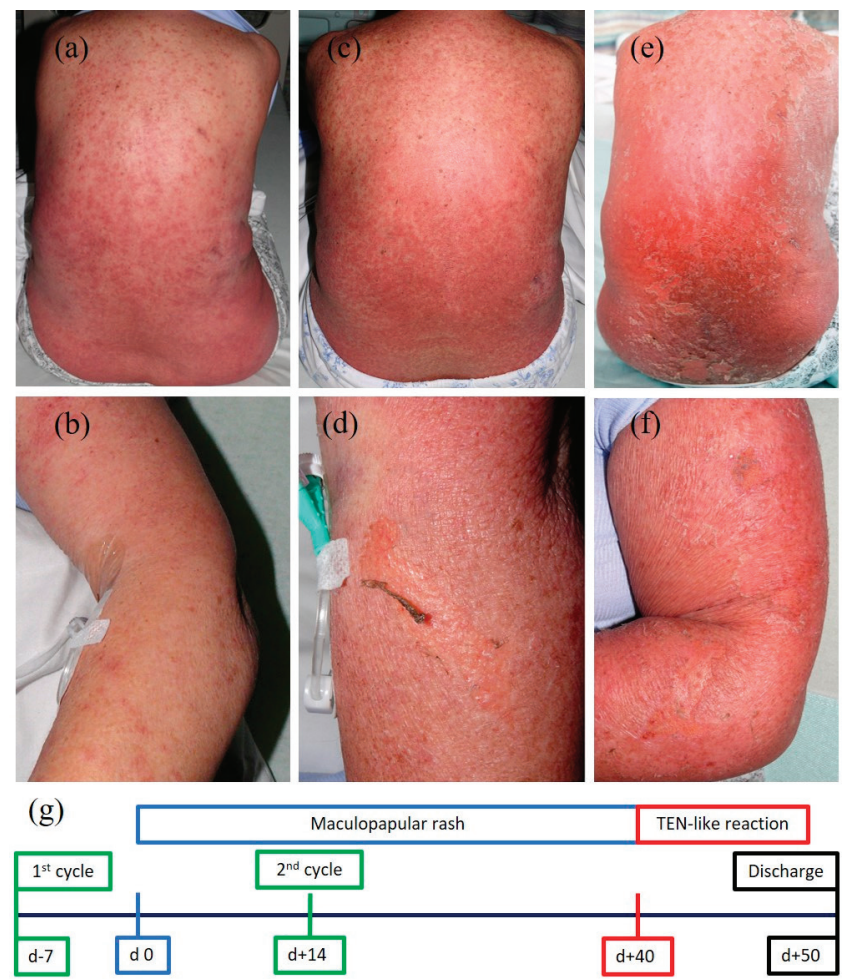

Figure 1. Development of the rash and timeline. Maculopapular eruptions on back (a) and left forearm (b) 27 days after onset of the rash. Coalescing and spreading maculopapular eruptions on back (c) and left forearm (d) together with beginning denudation 40 days after onset of the rash. Progression to widespread denudation on back (e) and left forearm ( $\mathrm{f}$ ) over the next days. Schematic timeline of events (g) with onset of the rash on day 0 ( $\mathrm{d} 0$ ).

daily to be weaned off by $10 \mathrm{mg}$ every 7 days was initiated along with supportive measures. The patient could be discharged to the community in a stable condition 50 days after the initial rash. Chemotherapy was discontinued and palliative care was initiated due to the sADRs including the diagnosis of a TEN-like reaction.

\section{Discussion}

Cutaneous ADR, usually grade 1- and grade 2 rashes are commonly seen with pemetrexed use, whereas Piérard-Franchemont et al [2] highlighted the difficulties interpreting these reactions as they are frequently labelled using the unspecific term 'skin rash'.

It is debated whether pemetrexed exhibits a direct cytotoxic effect of on the keratinocytes or elicits an indirect immune response underlying the pathophysiology of the drug's associated cutaneous toxicity $[2,4]$. The so-called antifolate cytotoxic skin reactions can clinically resemble TEN [4].

Supplementation of folic acid and vitamin B12 is routinely provided 1 week prior to chemotherapy aiming to minimise its cytotoxic effects, whereas the administration of oral or intravenous dexamethasone does not seem to reduce pemetrexed-associated cutaneous ADRs [4, 5].

To the author's knowledge, five cases of TEN-like reactions associated with pemetrexed were described in the literature to date. Tummino et al [6] reported on the appearance of a TEN-like reaction 15 days after administration of the second cycle of pemetrexed given for a refractory non-mall cell lung cancer. Another case of a TEN-like reaction including mucosal lesions and haemorrhagic blisters was noticed after the 
second cycle of pemetrexed and cisplatin given for metastatic non-small cell lung cancer [7]. Bosch-Barrera et al [3] described a case of a TEN-like reaction after the second cycle of pemetrexed, given in conjunction with carboplatin and Vitamin B12 and folic acid supplementation. A case of pemetrexed and carboplatin-associated TEN-like reaction in a patient with non-small cell lung cancer and pre-existing Sharp syndrome occurring primarily in the previous radiation field was reported by Then et al [8]. Another case of a TEN-like reaction in association with pemetrexed and cisplatin, given in combination with gefitinib, manifesting after the first cycle was described by Huang et al [9]. All of these cases described a rapid progression of the TEN-like reaction and none of the reported TEN-like reactions had a fatal outcome.

Whether or not a chemotherapy-associated adverse drug reaction needs the discontinuation of the treatment depends on the severity of the reaction. Maculopapular eruptions < 30\% TBSA are considered grade 1 or 2 adverse events and do not usually lead to discontinuation of treatment. However, TEN-like reactions are regarded as grade 3 or 4 adverse events and require discontinuation [10, 11].

The patient discussed in this study developed a limited maculopapular eruption 7 days after the first cycle of chemotherapy. Therefore, the treatment was continued. After the second cycle, the rash progressed to a grade 4 event with large areas of denudation. The patient's other chemotherapy-associated sADRs required multiple other medications. The onset of the rash before initiation of these medications argues for a pemetrexed- and carboplatin-associated event. However, the development of a second adverse reaction to one of these medications leading to the TEN-like reaction cannot be completely excluded. A Naranjo score of 6 was calculated, suggesting that the progressing maculopapular eruption was secondary to pemetrexed and carboplatin exposure [12].

\section{Conclusions}

The presented case of a slowly developing TEN-like reaction case aims to raise awareness about the finding that a maculopapular rash associated with pemetrexed and carboplatin can possibly progress into a more serious ADR. This should be taken into consideration when deciding on the continuation of the provoking treatment.

\section{Acknowledgments}

The author would like to thank L Goossens BSc, Senior Medical Photographer, for her service.

\section{Conflicts of interest}

The author declares that he has no conflicts of interest.

\section{Funding statement}

The author did not receive funding for publication of this manuscript.

\section{References}

1. Schwartz RA, McDonough PH, and Lee BW (2013) Toxic epidermal necrolysis: part II. Prognosis, sequelae, diagnosis, differential diagnosis, prevention, and treatment J Am Acad Dermatol 69 187.e1-e16 quiz 203-204 https://doi.org/10.1016/j.jaad.2013.05.002 PMID: 23866879

2. Piérard-Franchimont C, Quatresooz P, and Reginster MA, et al (2011) Revisiting cutaneous adverse reactions to pemetrexed Oncol Lett 2 769-772 [https://doi.org/10.3892/ol.2011.352] PMID: 22866124 
3. Bosch-Barrera J, Gaztañaga M, and Ceballos J, et al (2009) Toxic epidermal necrolysis related to pemetrexed and carboplatin with vitamin B12 and folic acid supplementation for advanced non-small cell lung cancer Onkologie 32 580-584 https://doi.org/10.1159/000232315 PMID: 19816075

4. Pierard-Franchimont $C$, Lesuisse $M$, and Humbert $P$, et al (2012) Toxic epidermal necrolysis and antifolate drugs in cancer chemotherapy Curr Drug Saf 7 357-360 https://doi.org/10.2174/157488612805076543 PMID: 23373549

5. Clark SK and Anselmo LM (2019) Incidence of cutaneous reactions with pemetrexed: comparison of patients who received three days of oral dexamethasone twice daily to patients who did not J Oncol Pharm Pract 25 1645-1650 https://doi.org/10.1177/1078155218804869 PMID: 30319062

6. Tummino C, Barlesi, and Tchouhadjian C, et al (2007) Severe cutaneous toxicity after Pemetrexed as second line treatment for a refractory non small cell lung cancer Rev Mal Respir 24 635-638 https://doi.org/10.1016/S0761-8425(07)91133-X PMID: 17519817

7. Scheinpflug K, Menzel C, and Koch A, et al (2012) Toxic epidermal necrolysis related to cisplatin and pemetrexed for metastatic nonsmall cell lung cancer Onkologie 35 600-603 https://doi.org/10.1159/000342671 PMID: 23038233

8. Then C, von Einem JC, and Müller D, et al (2012) Toxic epidermal necrolysis after pemetrexed and cisplatin for non-small cell lung cancer in a patient with sharp syndrome Onkologie 35 783-786 https://doi.org/10.1159/000345109 PMID: 23207626

9. Huang JJ, Ma SX, and Hou X, et al (2015) Toxic epidermal necrolysis related to AP (pemetrexed plus cisplatin) and gefitinib combination therapy in a patient with metastatic non-small cell lung cancer Chin J Cancer 34 94-98 https://doi.org/10.5732/cjc.014.10151 PMCID: 4360078

10. Sibaud V, Lebœuf NR, and Roche H, et al (2016) Dermatological adverse events with taxane chemotherapy Eur J Dermatol 26 427-443 [https://doi.org/10.1684/ejd.2016.2833] PMID: 27550571 PMCID: 5526115

11. Common Terminology Criteria for Adverse Events (CTCAE) Version 4.0 [http://www.hrc.govt.nz/sites/default/files/CTCAE\%20 manual\%20-\%20DMCC.pdf] Date accessed: 20/10/2019

12. Naranjo CA, Busto U, and Sellers EM, et al (1981) A method for estimating the probability of adverse drug reactions Clin Pharmacol Ther 30 239-245 [https://doi.org/10.1038/clpt.1981.154] PMID: 7249508 